\title{
The Design and Implementation of Real Time Encoder System
}

\author{
O-Byung Kwon \\ Division of Multimedia Engineering, College of Engineering, SungKyul University \\ shesthemen@naver.com
}

\begin{abstract}
This paper designed and implemented a next-generation solution for the Internet Broadcasting system applying FlashVideo. Compressing HD Class indges in real time currently being broadcast in the field, this is a system that can service VOD as well as Internet live broadcasting, which is a solution that supports the operation of Internet Live broadcasting, VOD service and UCC service easily onlime. It is a system that supports real time streaming of MPEC4 and WMV images on the Internet or Smartphone by compressing camera and VOD images using $\mathrm{H} 264$ Codec inreal time, which is first, the first real time encoder system in Korea with the real time recording feature of camera images and a software product that supports the up-to-date Codec technology suitable for the Web and Smartphone environments. Second, it is a wo-way Internet broadcasting system that can play videos with an MP4 player and support user chatting and customizing. Third, the Contents Management System (CMS) feature can service streaming of video contents and lecture management contents in real time through Android phone or iPhone.
\end{abstract}

Keywords: real timen internet broudcasting, HD (High Definition), flash video, compression

\section{Introduction}

With the rapid spread of the distribution of the Internet, a new concept of broadcasting media combining communication with broadcasting and sending out programs through the Internet appeared since the end of the 1990s, the Internet broadcasting is leading the revolutionary change in the domestic broadcasting environment.

In the early/phase of its launching, with poor quality broadcasting due to its poor production, it did not attract any special attention, but in the 2000s, as its potential ability that exceeded sky wave broadcasting: e.g., viewers can enjoy the broadcasts beyond time and space vas recognized, so including existing sky wave broadcasting, large online businesses continue to jump into the Internet broadcasting business.

Judging from the current speed of development, by 2020, the number of professional Internet broadcasting companies will be more than 2,000 , so that it is predicted that it will become the darling of the 21 st century new media industry, and the Internet broadcasting that shows a mighty power like this unlike existing sky wave broadcasting, produces and relays contents exclusively for the Internet and attracts attention as a new medium with infinite possibilities in that it takes its range of visibility of global netizens as well as its own nation.

The most characteristic advantages can be mentioned as follows: First, the maximum strength of the Internet broadcasting is that unlike sky wave broadcasting, it does not have any restriction on the number of channels and time of broadcasting, so the users can watch "the program they want" at "the time they like" in the two-way broadcasting type. Second, it 
deals with the area ground wave broadcasting has not been able to deal with due to viewer ratings or conditions of production and can target the niche market. In other words, it can exhibit differentiated media characteristics to its abilities. Third, it can provide customized broadcasting: providing the programs the viewers demand. It is forecasted that the number of small Internet broadcasting companies will gradually increase with its merit that individuals can operate just with simple equipment.

Thus, this article attempts to support easy operation of the Internet Live Broadcasting, VOD service and UCC service online with a system that can provide VOD service as well as Live Internet VOD by compressing HD videos in real time, compressing camera and VOD images using H264 CORECODEC in real time by implementing a system supporting MPEC4 and WMV videos on the Internet and Smartphone in real time streaming so that save VODs and transmit Live broadcasts in real time using the Web and Smartphone in-order to reduce traffic costs and solve security issues, to especially change the field of e-Learning rapidly expanding on the Web [1-3].

\section{Related Studies}

This Chapter will describe the differences between existing broadcasting and Internet broadcast, the transmission methods of the Internet broadcast services, and the structures of the current Internet broadcast system and In ernet broadcast data transmission structure, and Comparison and analysis by transmission methods.

\subsection{Differences between the existing broadcasting and Internet broadcast}

Internet broadcast is a technology in which communication and broadcasting are combined, and recently, which is promoting the growth of Yelated Internet industry with the expansion of the distribution of ultra high-speed access network and network advancements such as Internet Data Center (IDC) and Gontents Delivery Network (CDN), etc. The Internet broadcast services provide various specialized services unlike existing terrestrial broadcasting and satellite broadcasting, which have the following characteristics: They are interactive or bidirectional, and while in existing broadcast media, the senders transmit contents to the customers unilaterally, in the Internet broadcast, bidirectional communication is possible between the senders and the recipients, and the senders can provide enhanced services accepting the customers requests through conversations with individual recipients. Second, the customers can demand the services types and characteristics they want to the senders with personalized sefvices and diversification of channels, and they can receive contents whenever they want. In aedition, they can choose and repeat contents as they like, and through this, customizing services can be provided. Also, the Internet broadcast provides infinite broadcasting channels unlike existing broadcast media, and the service providers can develop and distribute contents fitting the customer's characteristics and demands. Third, the Internet broadcast services linked to other Internet industries can connect to the other Internet industries like e-commerce easily through the enforcement of security and charge function, and promote the creation of profits of related Internet businesses. 


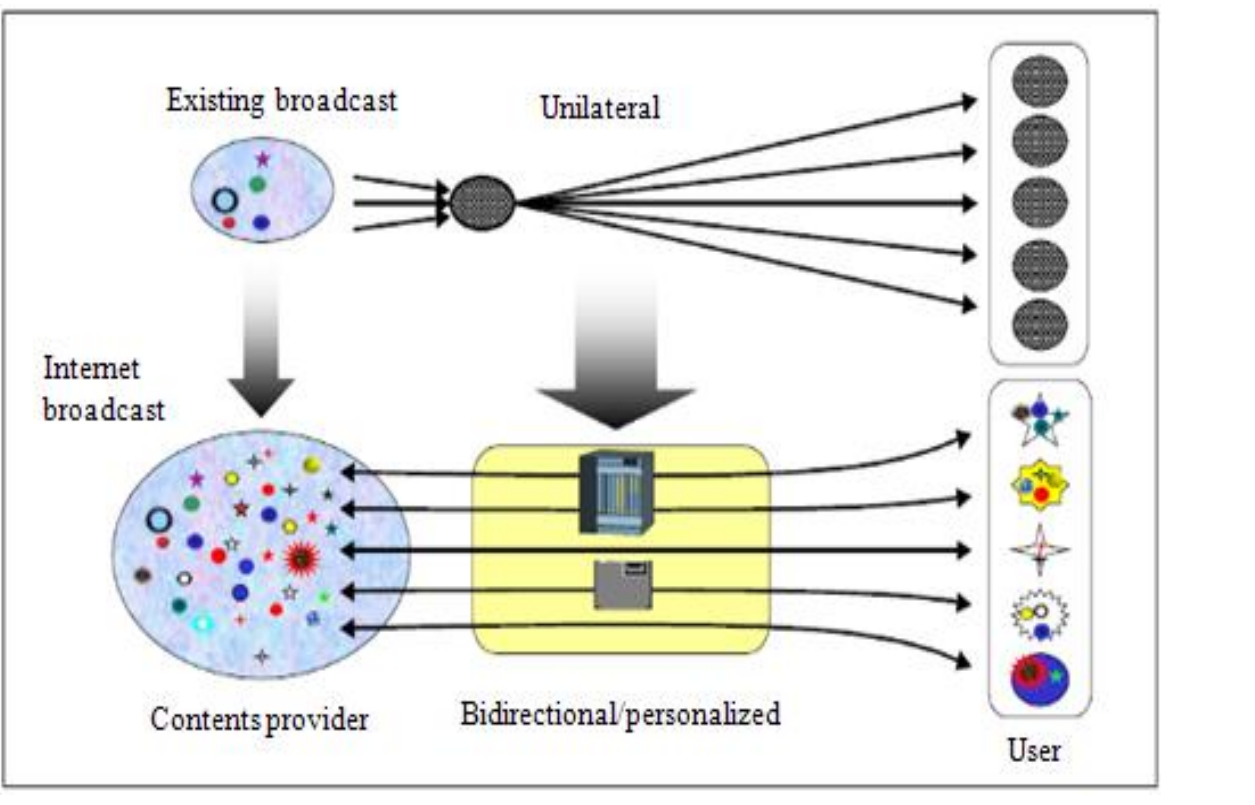

Figure 1. Differences between existing broadcasting and Internet broadcast

Figure 1 shows the differences betweenexisting broadcasting and Internet broadcast. The Internet broadcast can promote the growth of related Internet service industries by accepting the requests from the customers by bi-directionality, personalization and diversification of channels.

\subsection{Transmission metheds of Internet broadcast services}

The Internet broadcast technology can be used not only for the air channel broadcast but also for the Internet based e-commerde, video advertising such as product sales promotion and corporate PR, etc, variogs, events like real-time live broadcasting services, and various Internet services such as remote learning broadcasting by images and texts in parallel. The services provided throge the Internet broadcast can be divided broadly into two types by transmission methodas follows.

2.2.1. On-demard services: On-demand Internet broadcast services belong to a "Pull Service" model, in which contents are delivered on the request of the customers, and the kinds and types of contents and transmission speed may differ depending on their personal requests, and the customers can receive contents whenever they want. In addition, the contents can be controlled depending on their request: e.g. repetition, and the contents service providers can develop and distribute the contents according to the classifications and characteristics of the customers.

2.2.2. Live broadcasting services: The Internet live broadcasting services belong to a "Push Service" model, which come under the live relay of specific events not recorded broadcasting compared with TV broadcasting. This method is used to transmit the same contents to numerous customers simultaneously, and news and publicity media transmission services for numerous subscribers, too, fall under this.

\subsection{Structures of Internet broadcast system}

The Internet broadcast system consists of several broadcasting devices and servers. There are differences in the servers by the actual placement, but it consists of a Stream Server in 
charge of the Web Server and streaming transmission. In addition, an encoder creating or processing contents and a storage device storing generated contents are required. Figure 2 shows the structures of the Internet broadcast system. For the On-demand services, contents stored in a device can be sent to the customers through the Stream Server, and for the live broadcast, the contents created through the encoder can be transmitted to the customers through the Stream Server directly. If the customer accesses to the Web Server of a Contents Provider (CP) to request for related contents, the Web Server should choose a proper Stream Server by the live broadcast/On-demand services and the demand of contents and the selected Stream Server can transmit data to the customers via the Internet. The Internet broadcast system devices are already commercialized, and in particular, various products for streaming media transmission technologies such as Windows Media, Real Media, MPEG and Quick Time etc. have been launched and used.

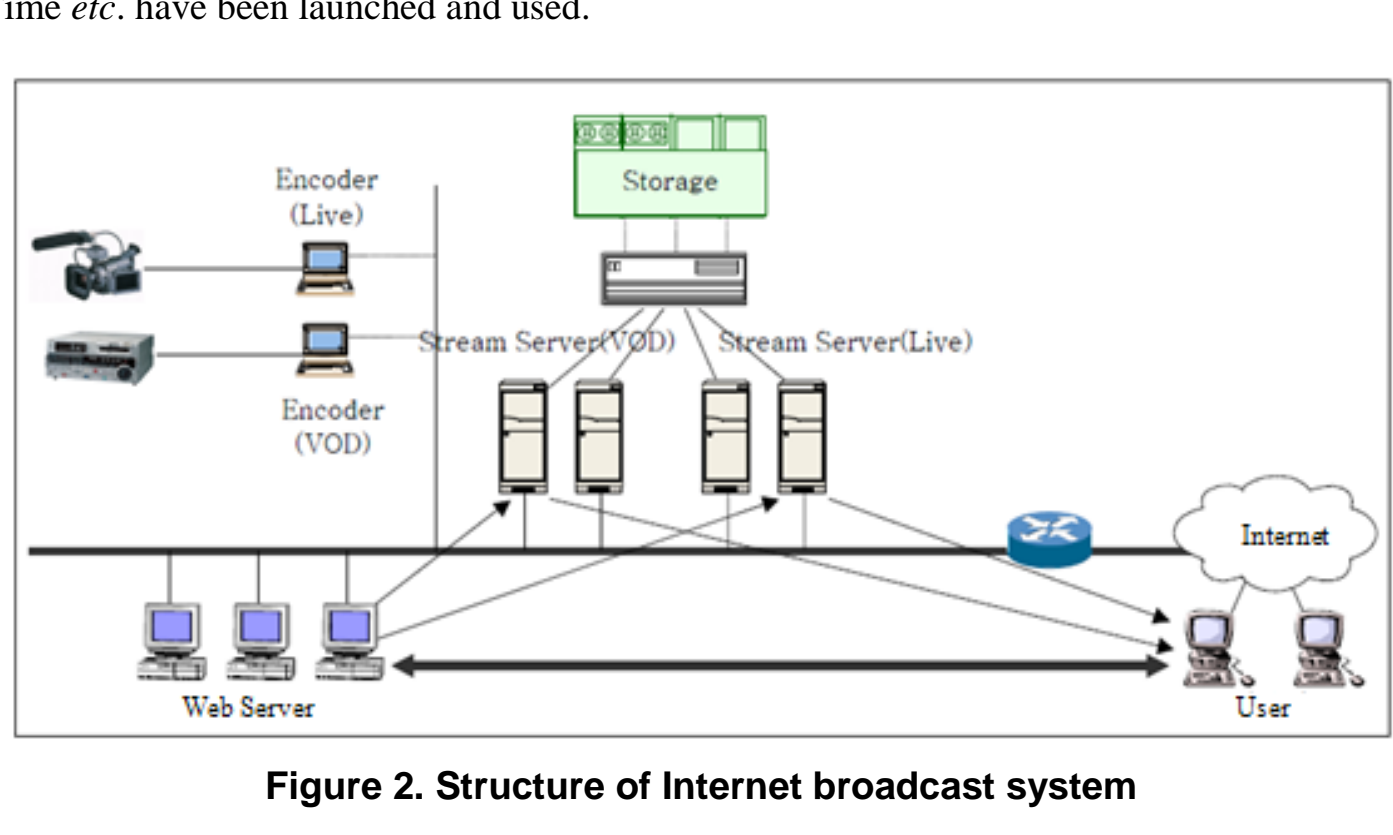

\subsection{Internet broadcast data transmission structure}

The Internet data transmission methods are classified broadly into Unicast and Multicast, and most Internet services are provided through a Unicast method based on TCP/IP or UDP/IP. Meanwhile, the Multicast technology developed for multilateral group communication'services like Internet broadcast, but it has not yet been widely popular. However recently, the affiliate application services markets such as Internet broadcast and distance learning etc. have been growing sharply, and it is expected that the Multicast technology will be widely used because of its better effectiveness and economic feasibility than those of Unicast. Especially, the Unicast transmission is suitable for the contents transmission specialized for individual customers like On-demand services, but live broadcast services should transmit the contents to a great many subscribers, so the Multicast transmission is required acutely for the effectiveness of the network resource. This Chapter describes transmission structures that can be used for the Internet broadcast contents transmission. Especially, it suggests alternatives in terms of the Unicast and Multicast transmission technologies, and compares and analyzes their advantages and disadvantages.

2.4.1. Unicast (Pure Unicast): This is a transmission method mostly used in the Internet broadcast services currently, and data packet is transmitted through TCP/IP or UDP/IP 
Unicast link between a sender and each individual recipient. As shown in Figure 3, through an individual Unicast link between the $\mathrm{CP}$ sender and each recipient customer, data are transmitted. Each recipient request for contents by accessing the CP's Web Server and the CP transmits the relevant contents using a Stream Server.

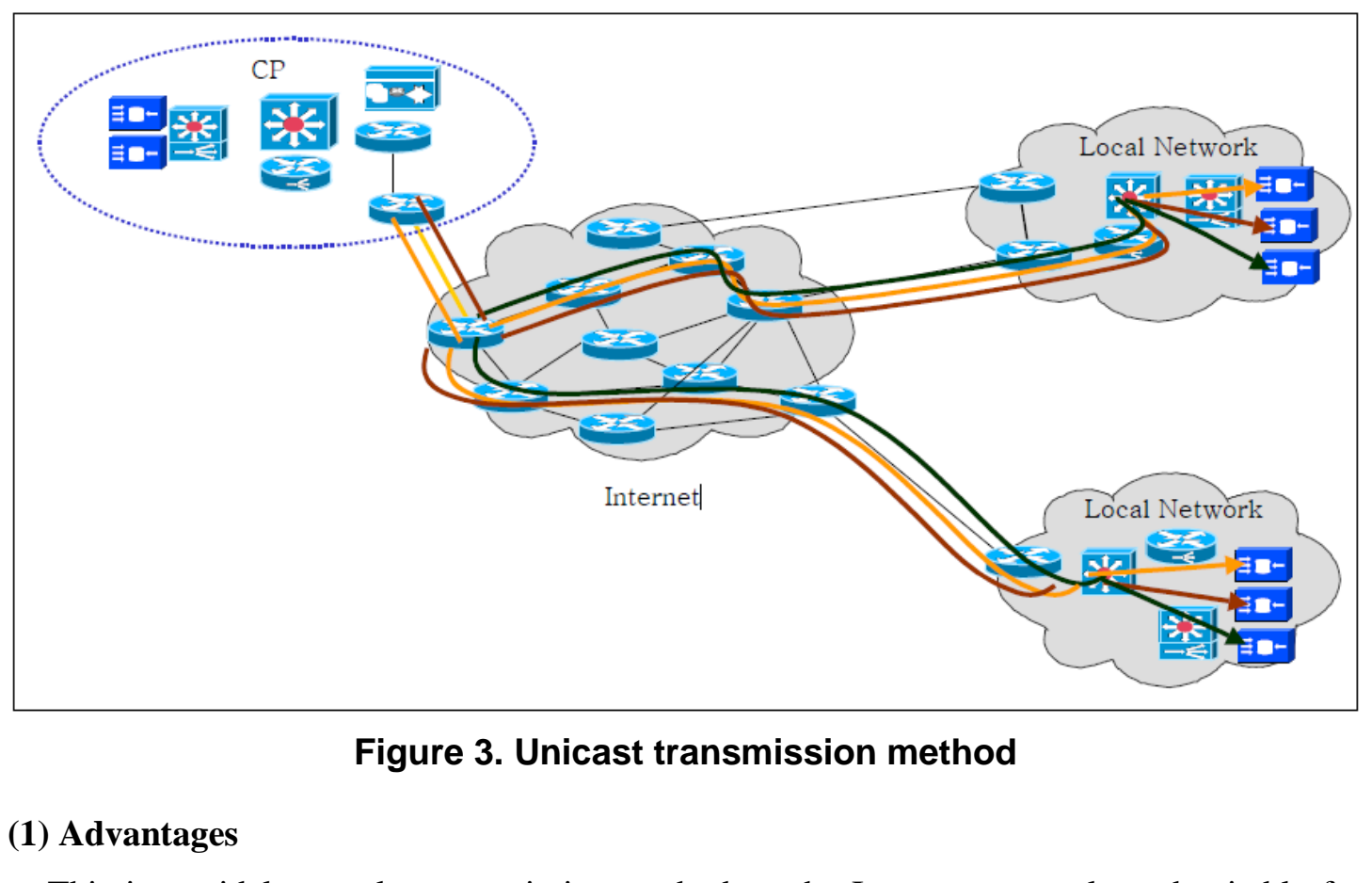

This is a widely popular transmission method on the Internet, currently and suitable for On-demand services providing individual customers with individualized services. Also, a direct link is set up between a CR and a customer, so the CP can easily get access information, contents use information and traffic statistical data of individual customers, so it is advantageous to establish fees and customer management policy.

\section{(2) Disadvantages}

Transmission delays and data losses may increases with changes in network environment such as partial congestion in the Internet network, according to which the so-called "MiddleMile" issue may occur: The data transmission quality is rapidly degraded. In addition, Unicast link is set up as many numbers of simultaneous log-ins, so a lot of network bandwidth is demanded, and there is a great load on the transmission Server system. Thus, by a CP's transmission system capacity and network link bandwidth, the number of simultaneous logins is considerably limited. It has a lot of limitations in applying to the Internet broadcast services and live broadcast services consisting of a large number of groups.

2.4.2. Relay Server-based Unicast Relay: This is a method of relaying a CP's transmission traffic to a recipient, in which a Relay Server is located for area or ISP domain. A Unicast link is used from the CP to the Relay Server and from the Relay Server to a recipient customer. While the transmission data goes through the Relay Server before reaching the receiving host, network upper layer "transport layer or application layer" function should be carried out, so it is also called "application layer Multicast" method. Figure 4 shows an example of the Relay Server-based Unicast Relay transmission method. The transmission 
traffic is transmitted to each Relay Server by Unicast, and individual customers receive the Internet broadcast data stream through a Unicast link from the Relay Server in geographically nearby locations. The CDN technique to which recently attention is paid is a typical example of the "application layer Multicast" method. A CDN transmission service provider installs a CDN local Server in each area of the network, a recipient is allowed to receive the Internet broadcast stream from a CDN Server located close not from a long range of a $\mathrm{CP}$ so that the so-called "Middle-Mile" issue of the Unicast transmission method can be solved. With the emergence of the CDN technique, a new business field of "CDN transmission business" is settling and several companies at home and abroad like Cisco Systems are developing and selling related solution products. In the CDN transmission business, Inktomi and Akamai (foreign firms), and KT and CDNetworks (domestic firms) are currently participating. Besides the CDN technique, a method of implementing Multicast function in application layer" is proposed. "Your Own Internet Distribution (YOID)" project in some academics in the United States studies a method of forming a logical tree betreen receiving hosts participating in a certain group and attempting Unicast relay between individual hosts along the tree. In the meantime, in Korea, companies such as EGC\&C and RJ Z Communications developed techniques of implementing Multicast transmission function in some receiving hosts by software not using a Multicast router in the network and are leading the market of the related fields.

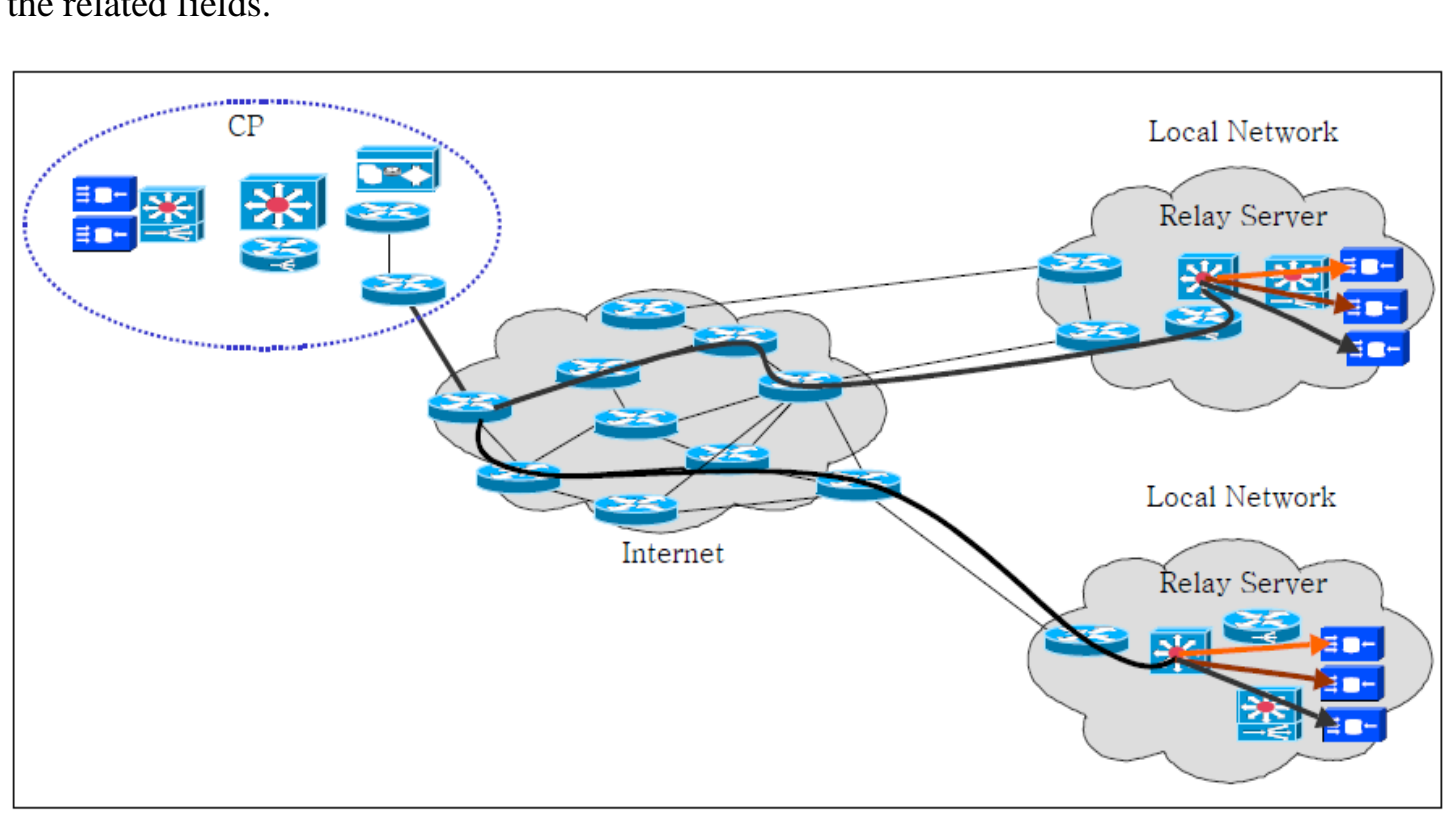

Figure 4. Relay Server-based Unicast Relay transmission method

\section{(1) Advantages}

The local Server-based Unicast Relay method is efficient in terms of use of network bandwidth and transmission system resource compared to the existing "pure Unicast" method. Also, from the position of customers, instead of setting up a link in a long range of a CP penetrating the Internet backbone to receive contents, they receive data from a Relay Server close to them, so the quality of the data transmission can be enhanced. This method is suitable for On-demand services. For a CDN, each CP distributes the contents to several CDN Relay Servers in advance. If a customer requests for contents transmission through a Web Server, 
the CP finds the optimal CDN Relay Server located near the customer, responds to the customer, and the customer accesses to the relevant Relay Server to receive the contents.

\section{(2) Disadvantages}

There are some problems in providing live broadcasting services by a CDN transmission structure. While live broadcasting services are characterized by numerous simultaneous logins and seamless transmission of real-time data, the CDN basically depends on Unicast Relay transmission, so if the number of simultaneous log-ins sharply increases, there is a limitation of its coping ability. In addition, while live broadcast stream reaches from a CP to a recipient, it should go through a conversion process: [network layer $\Leftrightarrow$ transmission/application layer $\Leftrightarrow$ network layer] in the CDN Relay Server. In the CDN method, contents distribution is performed by the $\mathrm{CP}$, but the actual transmission of contents is in charge of the CDN Relay Server, so compared to the pure Unicast method, the $\mathrm{CP}^{\mathrm{C}} \mathrm{s}$ contents and customer management function gets weaker, and all contents control, distribution, and management in the CDN network are done by the CDN transmission service provider, so the dynamics structure between "the $\mathrm{CP}$ and the $\mathrm{CDN}$ service provider" may develop centering around the $\mathrm{CDN}$ service provider to some extent.

2.4.3. Multicast (Pure Multicast): The Multicast method assumes that Multicast function is distributed to the Internet network. CP generates a Multicast session through a Web Server and notifies customers of session information needed to receive Multicast data. The customers get related information from the CP\%s Wéb Server, subscribe to the Multicast session and set up a Multicast link in the network. Throughout the course of these, a Multicast tree is formed between the network routers. As shown in Figure 5, data transmitted by the CP are delivered to each customer through the Multicast tree of the network. No separate Relay Server is not used.

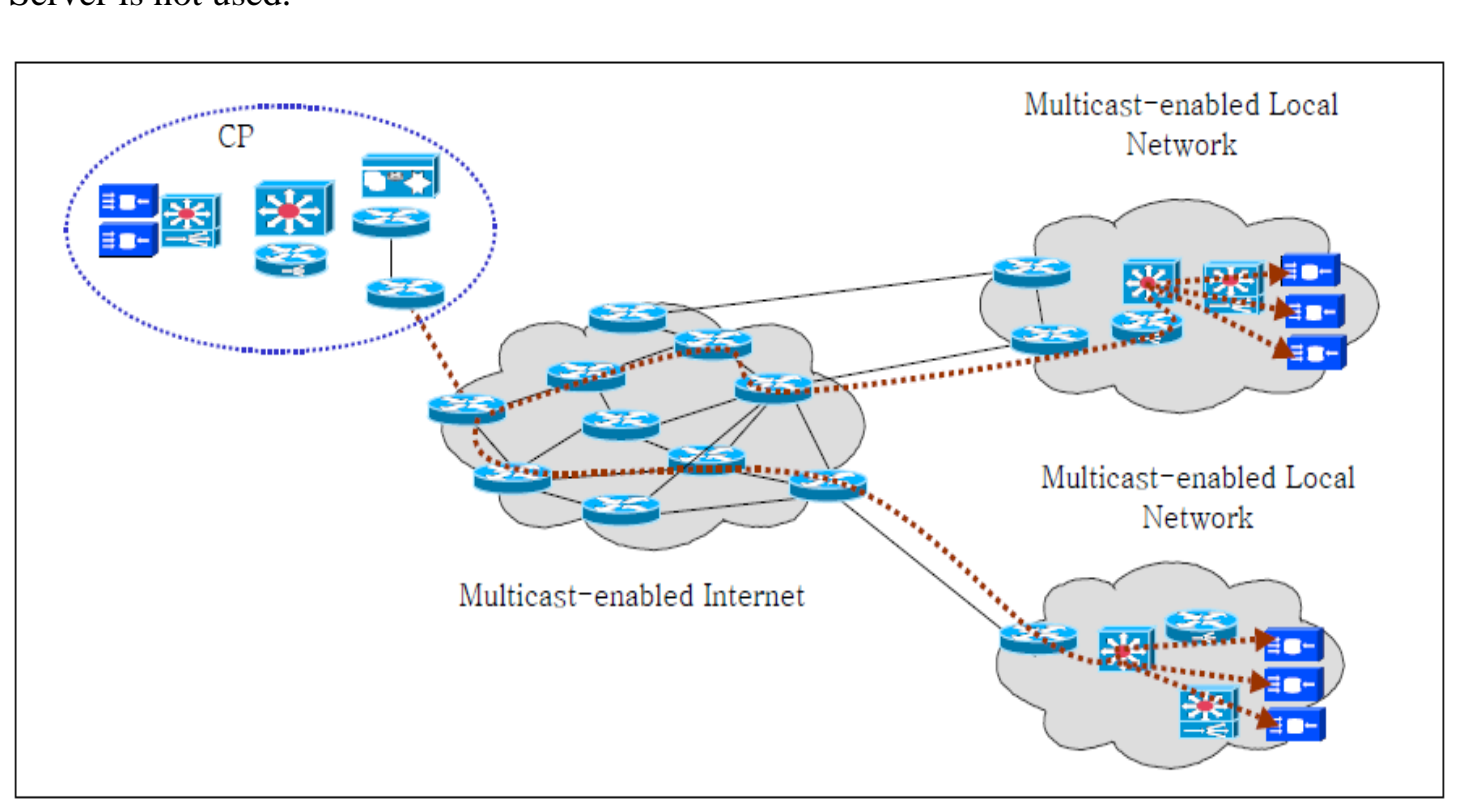

Figure 5. Multicast transmission method

\section{(1) Advantages}

IP Multicast technique is a standard technique sufficiently matured through research and development for 20 years, and compared to the Unicast method, it is very efficient in terms of 
use of network resource and transmission. Most common routers already have built-in Multicast function, so an ISP can use it once the formation of a related revenue model becomes concrete, The Multicast method is a transmission method very suitable for Internet live broadcasting services with numerous simultaneous log-ins, and theoretically, it can be delivered to infinite recipients. In addition, since all packet transmissions to the recipients are done at the network layer, the packet transmission time is very short.

\section{(2) Disadvantages}

This is suitable for live broadcasting services while it is not suitable for On-demand broadcasting services providing contents and quality specialized for individual customerse In Korea, some ISPs have introduced Multicast, but it has still not been introduced to many ISP networks. Especially, whether to support Multicast function in substructure of transmission such as DSL (Digital Subscriber Line) and cable modem has not yet been verified. Application of Multicast within an ISP network is relatively easy while Multicast transmission technique between different ISPs has still a lot of problems to solve.

2.4.4. Relay Server-based Multicast: Multicast dist ibution is easy in ISP network while it is predicted that it will take some time for Multicast function to be distributed to the entire Internet within a few days, so a transmission structure mixing Unicast and Multicast wi he an effective alternative. In this method, in an Internet backbone network not supporting Nulticast, Unicast link including IX (Internet eXchange) is used while in an area network supporting Multicast, using a Relay Server, Multicast link to individual customers is used Especially, this method is very effective when CP and the customers belong to a different ISP domain. As shown in Figure 6, from the CP to the Relay Server of each area network, Unicast linkis used to transmit data while the Relay Server transmits data to the individual customers through Multicast link. The customers located in the area network access to the CP's Web server, analyze the location of the Relay Server and the support of Multicast, and subscribes to Multicast group with the Relay Server to receive data.

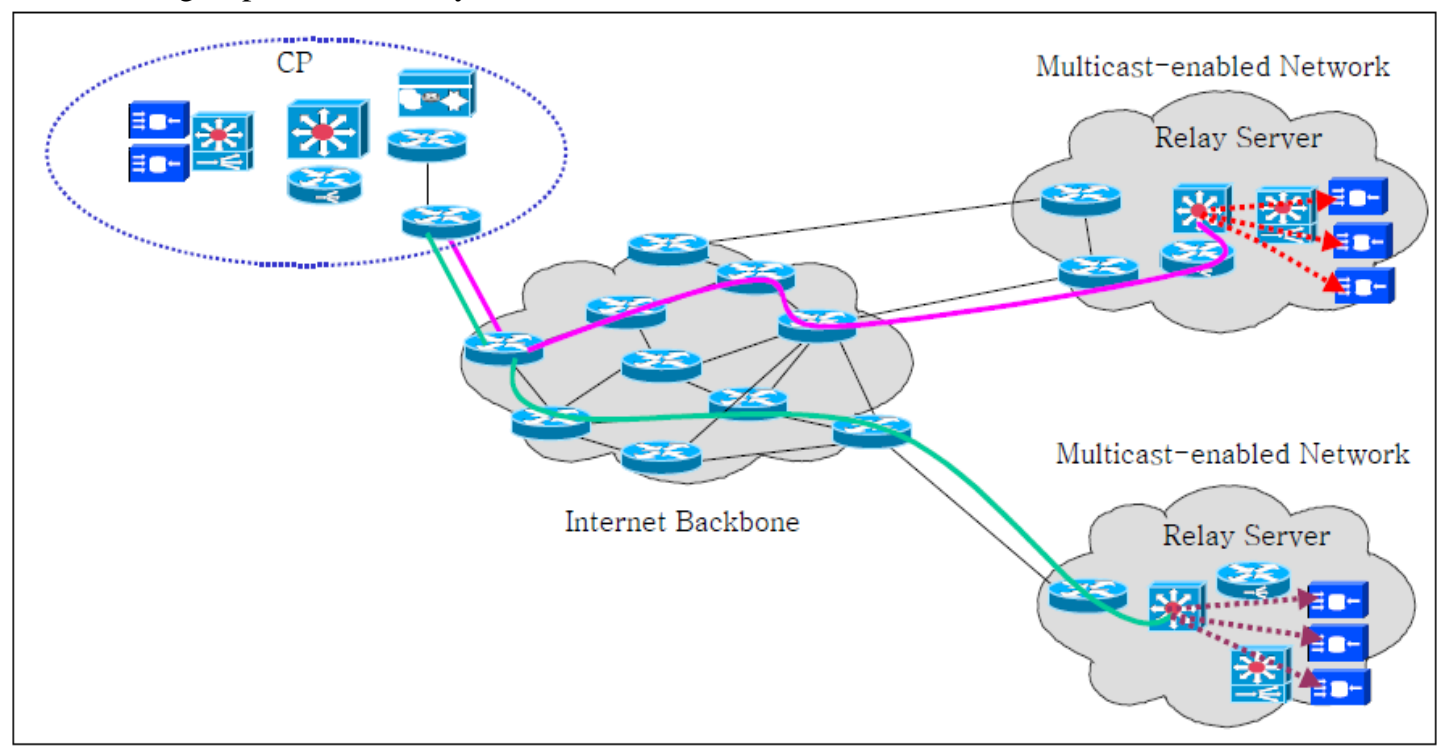

Figure 6. Relay Server-based Multicast transmission method

\section{(1) Advantages}

The above method is a mixed transmission method of Unicast and Multicast, which can be seen as a realistic alternative by the prediction of the introduction of Multicast. In addition, in an area network supporting Multicast, the effectiveness of use of the network resource using 
Multicast transmission can be promoted. Internet live broadcasting services are the representative target services. Also, if it is used in parallel with CDN transmission method at the local Relay Server, it can support both On-demand services and live broadcasting services. In this case, the local Server uses Unicast transmission for On-demand services demand while Multicast transmission for live broadcasting services.

\section{(2) Disadvantages}

For the seamless cascading of Unicast and Multicast transmissions in the local Server, detailed technical developments such as contents distribution management, Multicast group and session management are additionally required, but the development of the related techniques will be easily solved by using Multicast standard techniques developed till now [2].

\subsection{Comparison and analysis by transmission methods}

A comparison and summarization of merits/demerits and chatraeteristics of the four Internet data transmission methods described so far are like Table 1.The Unicas method is suitable for On-demand services while the Multicast or mixed methed is suitable for live broadcasting services. The effectiveness of use of network resource and processing capacity is high in the Multicast method. It can sharply increase in applying simultaneous log-ins or Multicast. At the contents and customer management level the Unicast ntethod is advantageous, but they also can be provided in the Multicase method through a separate group and session management technique. To sum up, to effectively support both On-demand services and live broadcasting services and increase the effectiveness and economics of use of resource, it is desirable to use the second and foutrthmethods in parallel.

\section{Table 1. Comparison of Internet Broadcast Data Transmission Methods}

\begin{tabular}{|c|c|c|c|c|}
\hline Characteristic & Unicast & $\begin{array}{c}\text { Relay Server- } \\
\text { based } \\
\text { Unicast }\end{array}$ & Multicast & $\begin{array}{c}\text { Relay Server- } \\
\text { based } \\
\text { Multicast }\end{array}$ \\
\hline Target services & On-demand & On-demand & $\begin{array}{c}\text { Live } \\
\text { broadcasting }\end{array}$ & $\begin{array}{c}\text { Live } \\
\text { broadcasting }\end{array}$ \\
\hline Resource use effe & Very low & Low & Very high & High \\
\hline Number of smultaneous log-ins & Very little & Little & A lot & Many \\
\hline Contents and customer management & Easy & $\begin{array}{l}\text { Relatively } \\
\text { easy }\end{array}$ & $\begin{array}{c}\text { Additional } \\
\text { management } \\
\text { needed }\end{array}$ & $\begin{array}{c}\text { Additional } \\
\text { management } \\
\text { needed }\end{array}$ \\
\hline Transmission services quality & Low & High & Very high & High \\
\hline
\end{tabular}




\section{System Design}

This chapter aims to describe the system implementation environment of internet broadcast solution, overall system design, video and web system design, video system design, and internet broadcasting solution diagram.

\subsection{System implementation environment}

\subsubsection{Video system implementation environment}

- DBMS : Oracle 9i or higher, My-sql, MS-sql

- Web Server : Apache-Tomcat-7.0.37

- Server OS : Redhat 9.0 or higher

- Development Language : PHP, Java, Dhtml, XML, Flash

- Video services environment : FMS (Flash Media Seryer) based/streaming services, encoding service

\subsubsection{Web system implementation environment}

- DBMS : Oracle 9i or higher, My-sql, MS-sql

- Web Server : Apache-Tomcat-7.0.37

- Server OS : Redhat 9.0 or higher

- Development Language : PHP, Java,Dhtml, XML,Flash

\subsubsection{Broadcasting System envifonment}

- Mobile Relay System : MS-500 (1 set)

- Composite Cable : CANARE Cable (Video/ntercom/Tally)

- Cable Reel : Doldolly AR-360

- 2 Units of Camera ; SONY DSR-PDL70

- Zoom Controller : LIBEC ZC-3DV

- Wireless Mic :SONY UWP-C

- Tripod : E[-71)

- Table Dolly System (tucluding a notebook installed on a table) : JC-9911

- Chroma Keyer System.DVK-100

- Chromatte : CKb 100

\subsection{Overall/System Design}

In Figure 7, actually, Front End consists of a Web Server or a streaming Server through L4 $\mathrm{S} / \mathrm{W}$ via the firewall through a Web browser, and the load dispersion Server disperses load to each Web Server by load balancing. The streaming Server provides streaming service of FLV file while the encoding Server converts WMV or other files to FLV to send to the streaming Server. Back End via L3 S/W consists a DB Server, security management Server, file Server, management Server and backup Server. 


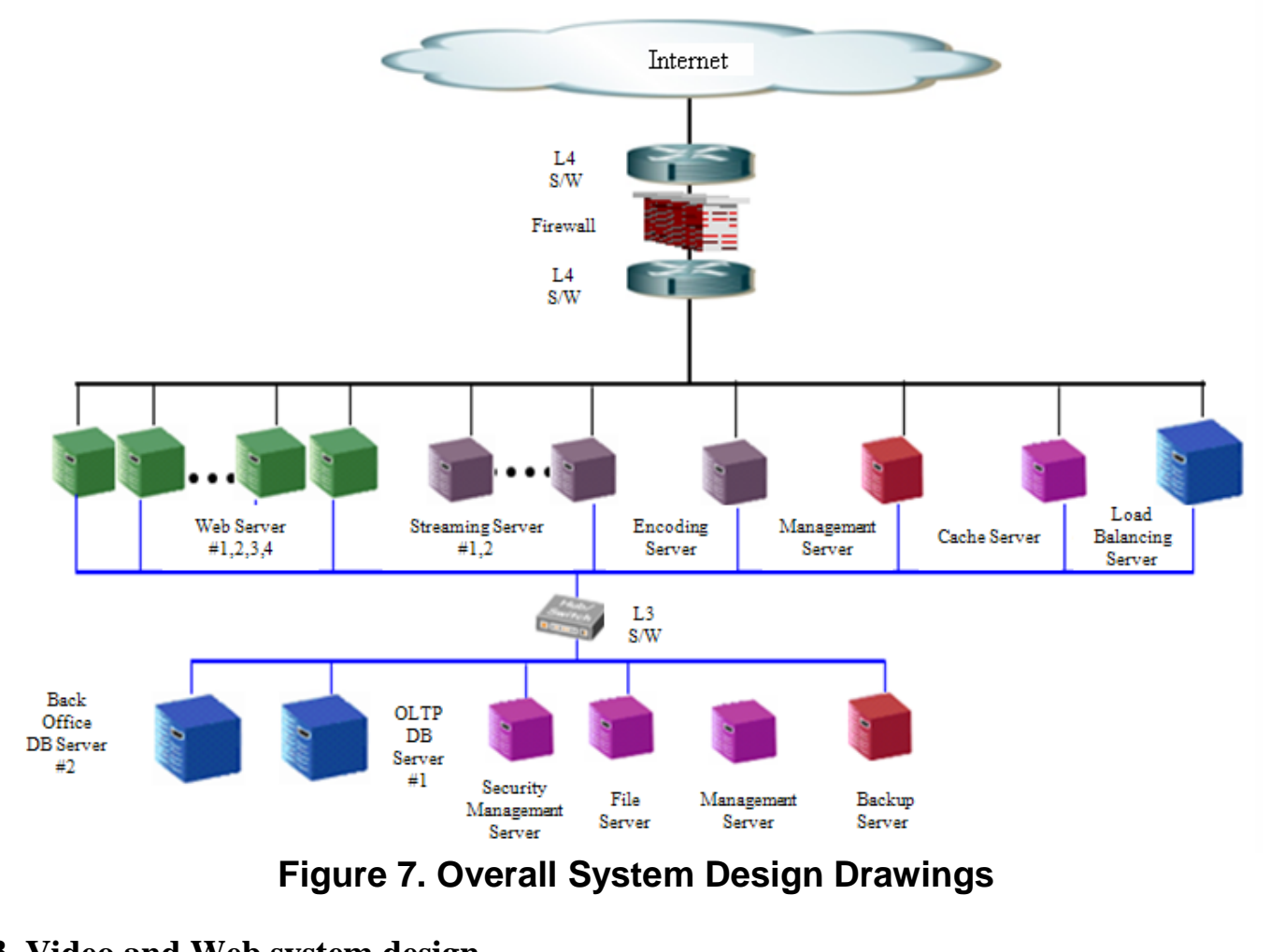

\subsection{Video and Web system design}

Figure 8 consists of a vide6 hosting Server, Web environment improvement Server, cache Server, load dispersion Server and Web Server, and the user can see video hosting, LMS and homepage services sent to the Web Server connected to the load dispersion Server, Web Server and the streaming Server of the video hosting Server, the DB Server and file Server of the Web environment improvement Server.

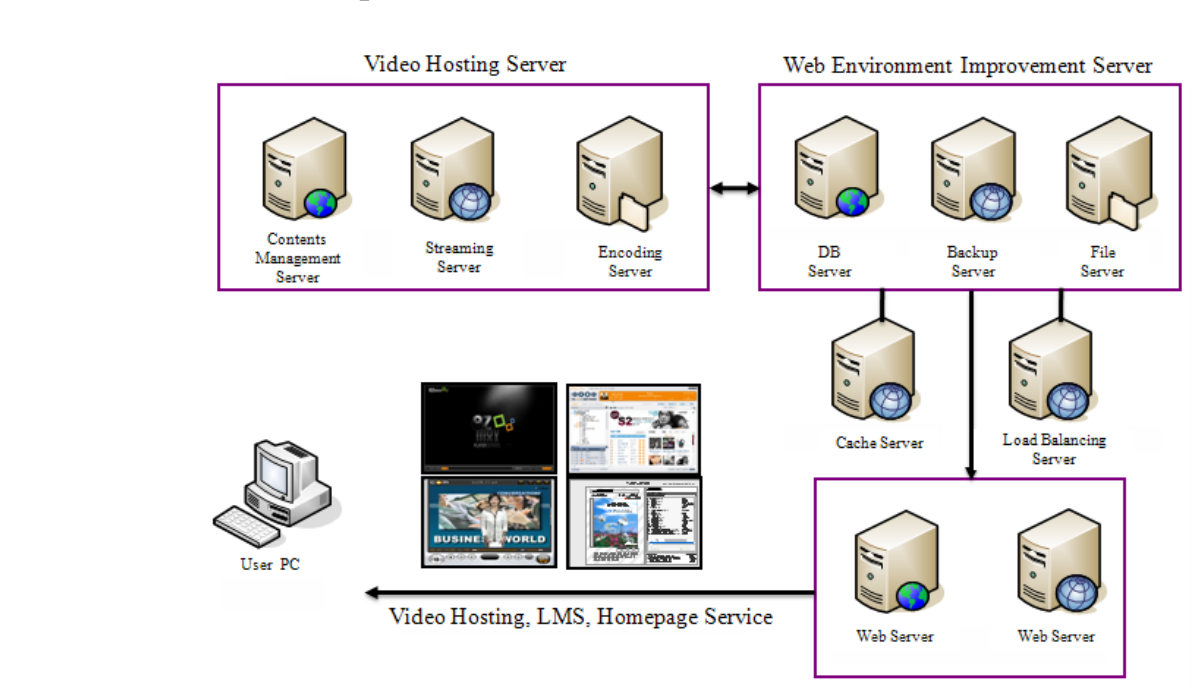

Figure 8. Video and Web system design drawings 


\subsection{Video system design}

Figure 9 shows that if the enterprise registers video, the contents management Server manages the video, manages and operates corporate logo, player size, storage capacity and traffic, and if it is an FLV file, it is sent from the streaming Server to the Web Server linked to the DB Server, and if it is a WMV or other files, the encoding Server converts it to FLV and sends it to the streaming Server, and then it is sent to the Web Server linked to the DB Server, and the user is provided with services simultaneously with the access to the Web [4-8].

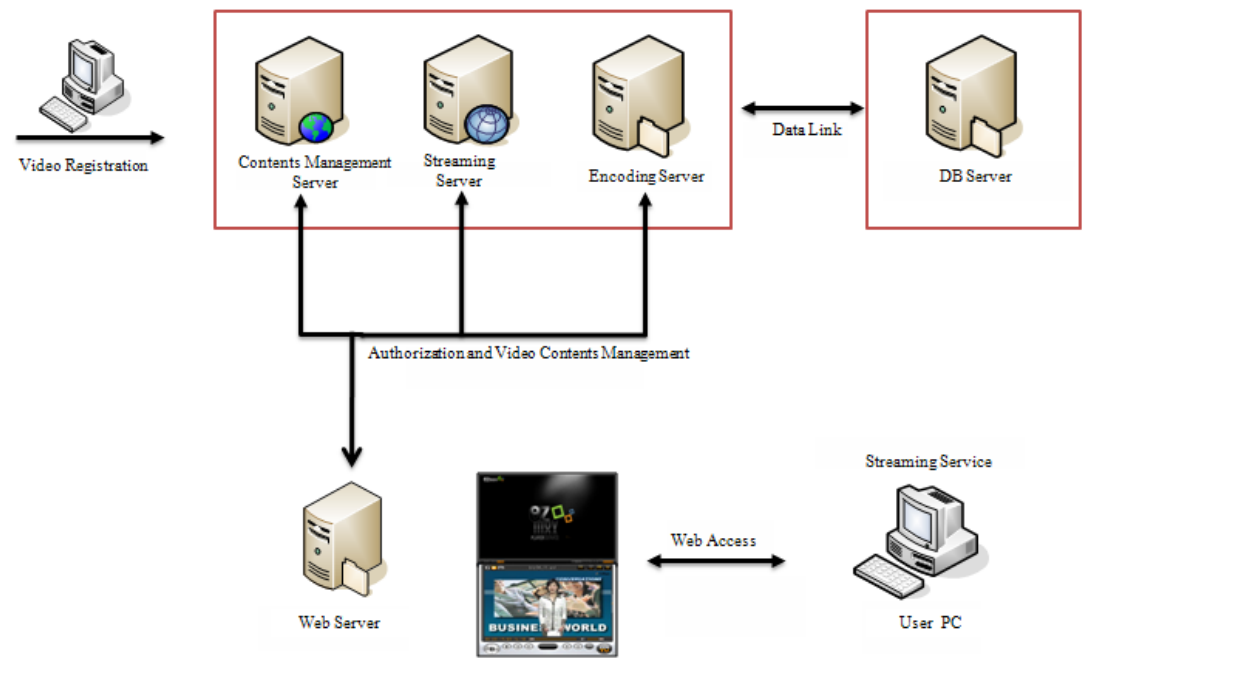

Figure9. Video Systèm Design Drawings

\subsection{Internet Broadeasting Solution Diagram}

Figure 10 shows a structure in which the system receives video inputs through HD cameras, saves the inpu video using real time encoding system (S/W), and the customers can see the saved video streaming on the Web in real time through a player, and in addition, it by organizing the video coming in through the cameras through a scheduler system by date and hour, so that it can transmit and operate the video to broadcasting companies in the form of IPTV.

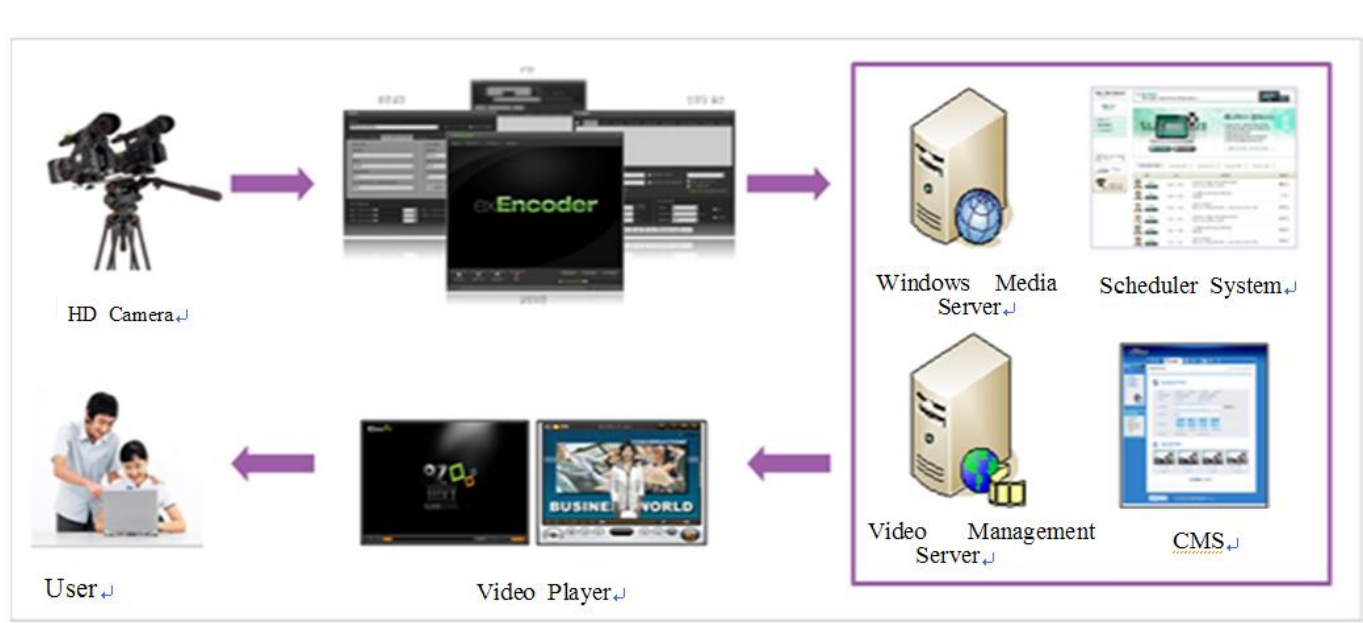

Figure 10. Internet Broadcasting Solution Diagram 


\section{Merits of Internet Broadcasting Solution}

First, it is the first real time encoder system in Korea with the real-time recording feature of camera images, supporting the up-to-date CORECODEC technology suitable for the Web and smart environments, which is not a hardware product but a software product.

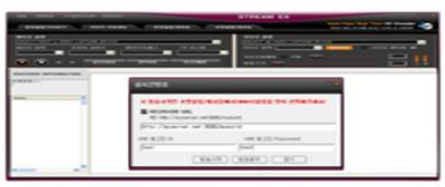

Video and audio settings

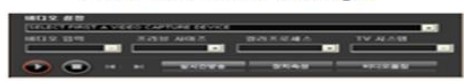

Video quality, compression codec and logo settings 1 .

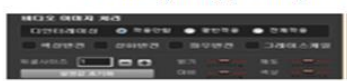

Figure 11. Real Time Encoder System
- Real time recording (encoding) while watching multiple camera images directly,

- Supporting dual recordings in various image formats (WMV, AVI and MPEG)
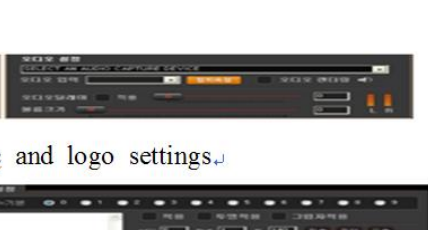

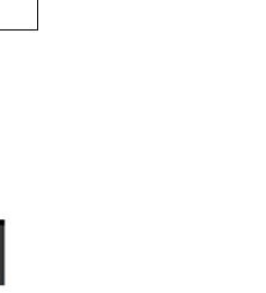



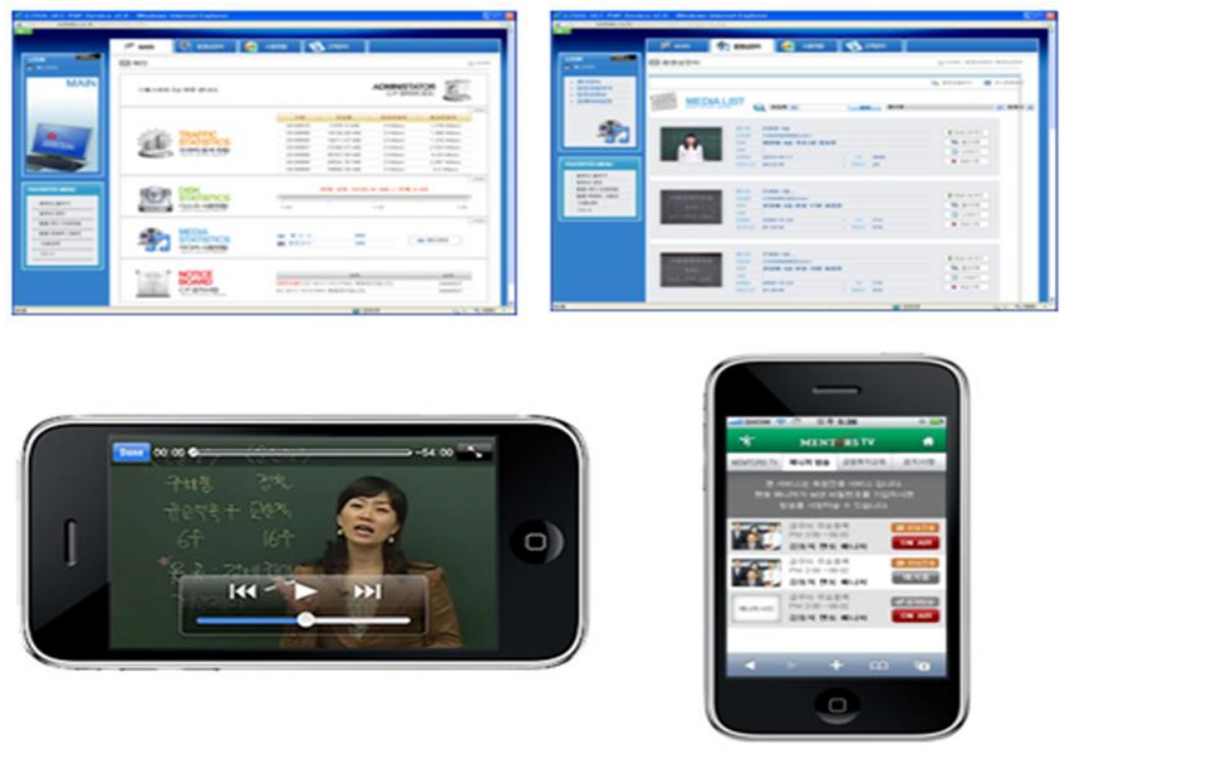

Figure 13. Contents Management System

\section{Conclusions and Future Work}

This is a system that can provide VOD service as well as live Internet VOD by compressing HD videos broadcast in the field in yeal time, for which a next generation Internet broadcasting solution that would support easy operation of Internet Live broadcasting, VOD service and UCC service online was designed and implemented. Everyone agrees that in the fluture, Internet broadcasting is a medium that will develop infinitely with the convergence between broadcasting and IT, but most Internet broadcasting companies run by indivicuals are small broadcasting companies, and they do not have any special basis for profits, so a pessimistic view might be suggested that they will disappear from the market within 2 or 3 years. Yet, it is clear that it is the eye of a typhoon in the future broadcasting market.

In the future, it is planned to construct a lot of information and data to contribute to the development of the Internet broadcasting system.

\section{References}

[1] Y. Y. Doo and C. E. Young, “ Effectiveness of the Flash Video on production of the E-Learnin”, Journal of the Korean society for contents, vol. 7, no. 4, (2007) April, pp. 192-198.

[2] S. J Koh, "New Trend on Internet Multicast Technologies", Journal of the Korea information and communications society, vol. 17, no. 9, (2000) September, pp. 168-187.

[3] H. D. Hwan, C. G. Seop and H. S. Yong, " Video Processing of the Multimedia Networking on the Compression Signal” , Journal of Korea Information and Communications society, vol. 26, no. 11, (2001) November, pp. 1908-1924.

[4] M. Park, O. B. Kwon, I. Kim and I. Choi, "Hankook Education Development Company", " Improve WMV and Existing FLV New Move Picture Solution EZ-MOV" , Proceedings of the Korea Multimedia Society Conference, vol. 11, no. 2, (2008) November, pp. 1-5.

[5] O. B. Kwon and H. C. Shin, " System Design and Implementation for New Move Picture Solution EZ-MOV Using FLV” , Journal of Korea Information Assurance Society, vol. 9, no. 2, (2009) June, pp. 79-84.

[6] G. H. Kwon and B. G. Joo, " Development of FLV Streaming Server” , Journal of Security Engineering, vol. 6, no. 1, (2009) February, pp. 39-46. 
[7] O. B. Kwon and H. C. Shin, " System Design and Implementation of FLV Move Picture Solution Based on IDC apply to Mini IPTV” , Journal of Korea Information Assurance Society, vol. 11, no. 4, (2011) September, pp. 11-17.

[8] O. B. Kwon, " The Design and Implementation for New Move Picture Solution EZ-MOV Using FLV” , International Journal of Advancements in Computing Technology, vol. 5, no. 12, (2013) August, pp. 491-504.

\section{Author}

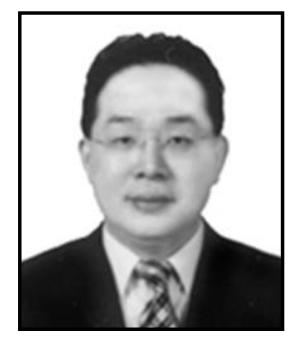

\section{O-Byung Kwon}

E-Mail : shesthemen@ naver.com

Website URL : http://www.sungkyul.ac.kr

Research Interest : Software Engineering, Multimedia Thformation processing, and Programming Languages.

He received Ph.D. in computer science from॰Dankoøk University, Korea in 2007. Since then, he has been with sungkyul University as a professor.

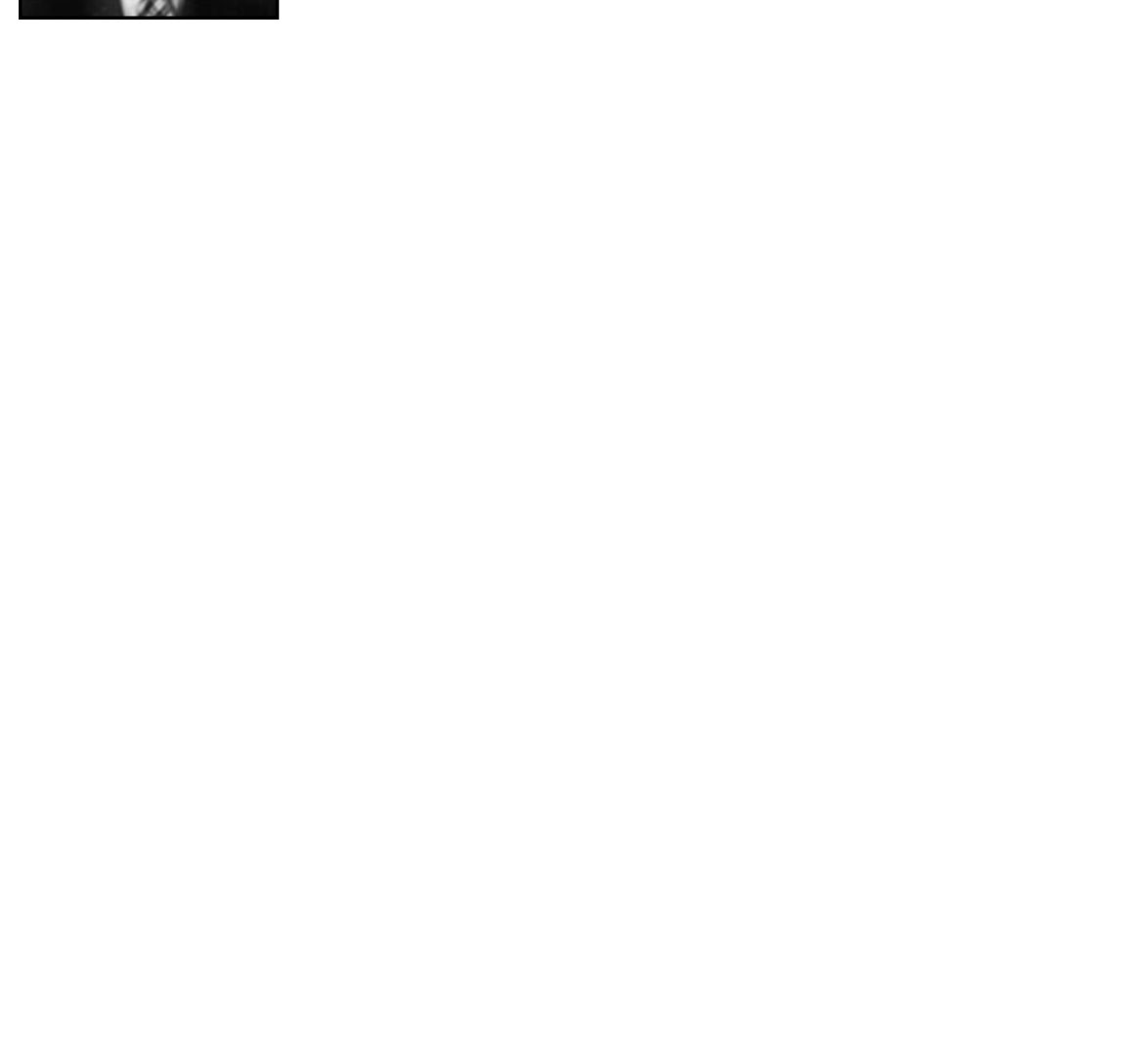


International Journal of Multimedia and Ubiquitous Engineering Vol.9, No.5 (2014)

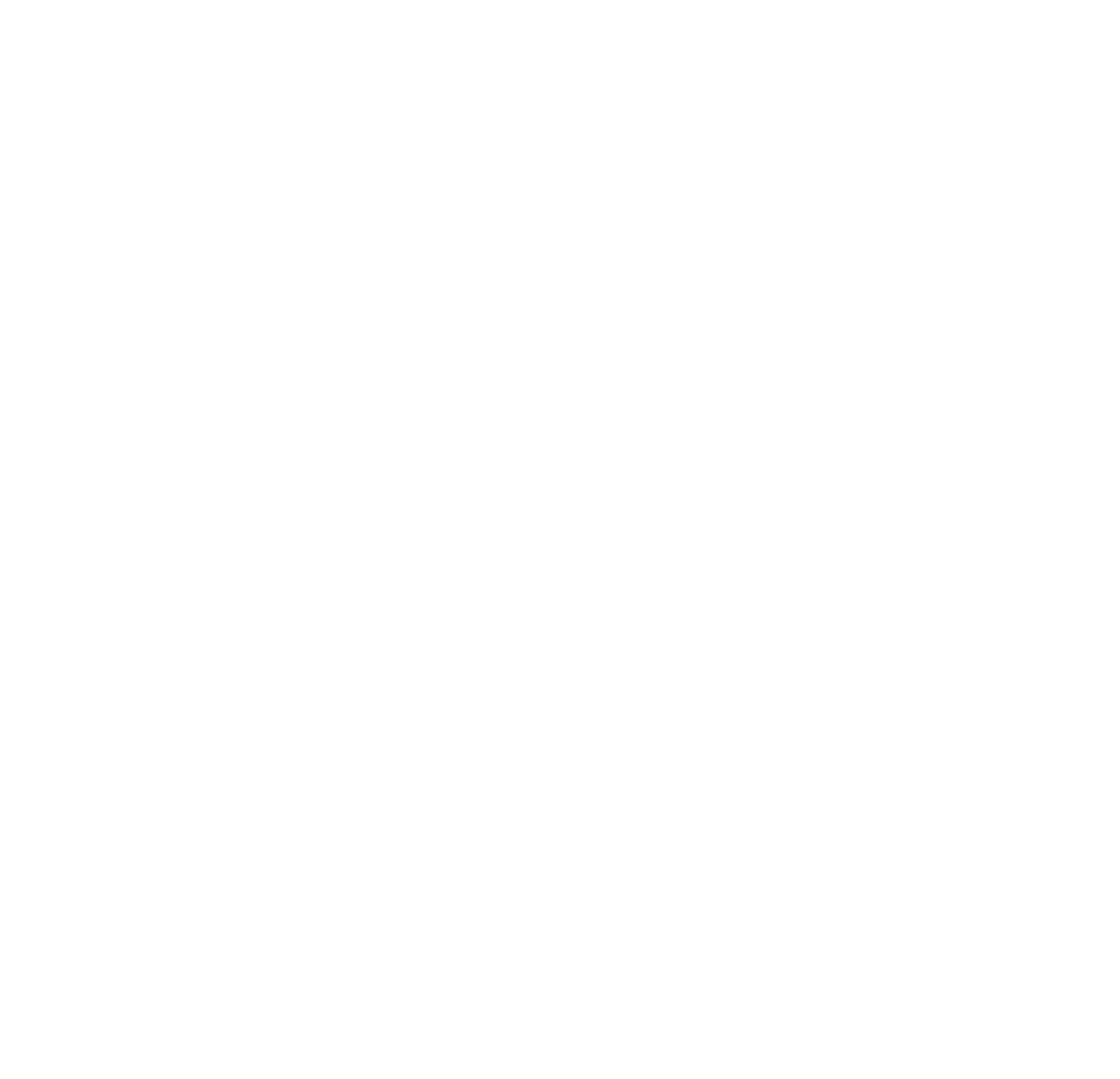

